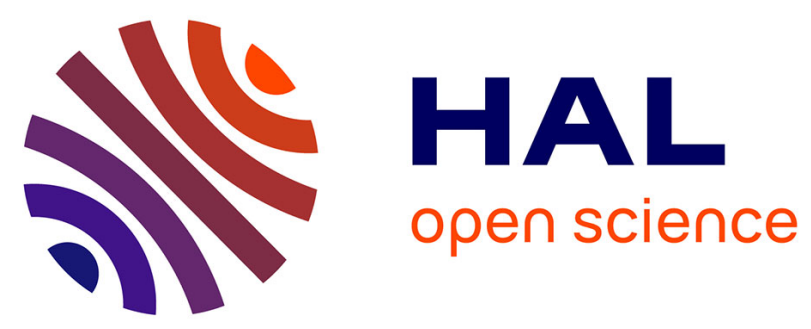

\title{
Optical patch antennas for single photon emission using surface plasmon resonances
}

Ruben Esteban, Tatiana V. Teperik, Jean-Jacques Greffet

\section{To cite this version:}

Ruben Esteban, Tatiana V. Teperik, Jean-Jacques Greffet. Optical patch antennas for single photon emission using surface plasmon resonances. Physical Review Letters, 2010, 104 (2), pp.026802. 10.1103/PhysRevLett.104.026802 . hal-00574380

\section{HAL Id: hal-00574380 \\ https://hal-iogs.archives-ouvertes.fr/hal-00574380}

Submitted on 4 Dec 2015

HAL is a multi-disciplinary open access archive for the deposit and dissemination of scientific research documents, whether they are published or not. The documents may come from teaching and research institutions in France or abroad, or from public or private research centers.
L'archive ouverte pluridisciplinaire HAL, est destinée au dépôt et à la diffusion de documents scientifiques de niveau recherche, publiés ou non, émanant des établissements d'enseignement et de recherche français ou étrangers, des laboratoires publics ou privés. 


\title{
Optical Patch Antennas for Single Photon Emission Using Surface Plasmon Resonances
}

\author{
R. Esteban, T. V. Teperik, ${ }^{*}$ and J. J. Greffet ${ }^{\dagger}$ \\ Laboratoire Charles Fabry, Institut d'Optique, CNRS, Université Paris-Sud, Campus Polytechnique, \\ RD 128, 91127 Palaiseau cedex, France
}

(Received 19 June 2009; revised manuscript received 27 October 2009; published 14 January 2010)

\begin{abstract}
Single photon sources can greatly benefit from specially designed structures that modify the properties of the photon emitter. Dielectric cavities are often discussed, but they require a compromise between the spectral width and Purcell factor. In this Letter, we introduce plasmonic cavities as promising alternatives. We first study how the emitter couples with the modes of such structures. We then show how a patch antenna configuration simultaneously presents a large Purcell factor, collection efficiency, and spectral width.
\end{abstract}

The design of a single photon source has been the focus of much recent work [1] for applications such as quantum key distribution. Many emitters currently available such as nitrogen-vacancy (NV) centers in diamond have typical lifetimes of $10 \mathrm{~ns}$ and emit with a broad angular pattern. For practical applications, it is desirable to emit in a given spatial mode and with an emission rate on the order of 100 ps to take full advantage of a mode-locked laser with a repetition rate of $1 \mathrm{GHz}$. A microcavity can be used to (i) increase the emission in a given spatial mode and thus the photon collection efficiency [2] and (ii) to reduce the emission lifetime taking advantage of the Purcell factor $F_{p}$ [3]. Yet, microcavities with a large Purcell factor have a large quality factor and thus a narrow bandwidth, so that they are not ideally suited for single photons emitters with broad emission spectrum. In this Letter, we will show that plasmonic nanoantennas are promising candidates to enhance the capabilities of single photon sources. Good photon collection efficiencies, and $F_{p}$ factors up to several hundreds with a bandwidth of tens of nanometers or more can be achieved with the plasmonic patch antenna introduced in this work. Since $F_{p}$ is proportional to the quality factor and inversely proportional to the effective volume of the electromagnetic mode, the key to large $F_{p}$ values over a significant bandwidth is strong spatial confinement of the resonant modes.

Examples of optical nanoantennas, i.e., antennas of nanometer dimensions working at optical frequencies, already considered for the modification of the emission include Yagi-Uda antennas [4,5] and dipoles [6], which in the latter case can be center fed [7]. Metallic disks with radius comparable to the wavelength, embedded in glass with refractive index $n_{g}$ and placed above a larger plane, are similar to classical patch antennas [8]. Such structures have been considered, for example, for infrared laser or terahertz sources [9]. These works studied guided modes (with wave vector parallel to the planes smaller than $2 \pi n_{g} / \lambda_{0}, \lambda_{0}$ being the vacuum wavelength), confined by metallic surfaces. Here, we will be interested in the exci- tation [10] of surface plasmons modes [11], which have a parallel wave vector larger than $2 \pi n_{g} / \lambda_{0}$. An alternative view of a thin metal slab is thus to consider it not as a component of a patch antenna but as a surface plasmon cavity, with the edges serving as reflectors and constructive interference leading to resonances. A similar perspective was taken in studies of nanowires [12], metal-insulatormetal (MIM) structures [13], and single [14] or coupled [15] nanostrips. Here, our goal is to optimize a solid state single photon source. We first analyze the simple case of a metallic disk to explore the physics of a surface plasmon cavity. We then introduce a patch antenna design that increases the field confinement due to the MIM configuration.

To characterize quantitatively the nanoantenna we use different figures of merit. The total decay rate $\Gamma_{T}$ is the inverse of the lifetime and directly relates to the local density of states, LDOS. More exactly, in the absence of intrinsic losses $\Gamma_{T} \propto \rho_{z}^{e}$, where $\rho_{z}^{e}$ is the projection of the electric component of the LDOS into the $z$ orientation of the dipolar emitter $[16,17]$. In a similar way, we use the expression $F_{p}=\Gamma_{T} / \Gamma_{0}$ for the Purcell factor of our structures, where $\Gamma_{0}$ is the value in homogenous glass without optical nanoantenna. $\Gamma_{T}$ can be split into radiative $\Gamma_{R}$ and nonradiative $\Gamma_{\mathrm{NR}}$ contributions $\Gamma_{T}=\Gamma_{R}+\Gamma_{\mathrm{NR}}$. The radiative yield $\eta=\Gamma_{R} / \Gamma_{T}$ describes the probability of photon emission by an excited quantum emitter. Here, we assume no intrinsic losses, $\eta=1$ in the absence of the optical nanoantenna. Finally, another important feature of a nanoantenna is the possibility to control the emission direction [18], quantified by the concept of directivity [8] $D(\theta, \phi)$ at a direction $(\theta, \phi)$ :

$$
\frac{P(\theta, \phi)}{P_{T}}=\frac{D(\theta, \phi)}{4 \pi},
$$

where $P(\theta, \phi)$ is the power per unit solid angle emitted in a given direction and $P_{T}$ is the total emitted power. $D(\theta, \phi)$ thus corresponds to the scattered intensity towards a given direction divided by the equivalent intensity for an iso- 
tropic source of the same total radiated power. $\int D(\theta, \phi) d \Omega /(4 \pi)=1$ by definition. Limiting the integral to a given solid angle $\Omega$ gives the fraction of photons $\beta$ that can be collected by a lens of corresponding numerical aperture, a critical parameter for single photon sources that we call collection efficiency.

We will consider spontaneous emission from a quantum emitter, such as a molecule, a quantum dot, or a NV center in a diamond nanocrystal, characterized by a given dipole strength. The emitter has no intrinsic losses and emits at a vacuum wavelength $\lambda_{0}$. If not otherwise mentioned, its position is $d=20 \mathrm{~nm}$ above the center of the surface of a silver disk of thickness $t=20 \mathrm{~nm}$, rotationally symmetric with respect to the $z$ axis and flat except at the rounded edges [Figs. 1(a) and 1(b)]. For simplicity, we use bulk values of the silver permittivity $\epsilon_{\mathrm{Ag}}$ [19] and ignore nonlocal effects [20]. A glass matrix of $\epsilon_{g}=2.25$ contains the complete system. The radius of the disk is $R=600 \mathrm{~nm}$, measured from its center to the furthest point in the boundary, and the quantum emitter dipole orientation is vertical, i.e., along the direction $z$ in the figure. Because of rotational symmetry, emission does not depend on $\phi$. We use a multiple multipole solver of the Maxwell equations $(\mathrm{MaX}-1)$ [21] to reveal how the electromagnetic response of the disks affects the spontaneous emission.

Figure 1 summarizes the results for the flat disks. The change in radiative decay rate $\Gamma_{R} / \Gamma_{0}$ shows up to five clear peaks at different wavelengths [Fig. 1(c)]. Figures 1(e2) and 1(f2) illustrate the field distribution for two of the peaks. Standing wave patterns are present, with the number of nodes depending on the wavelength, as expected for a cavity. The nonradiative decay rate $\Gamma_{\mathrm{NR}} / \Gamma_{0}$ in Fig. 1(d) shows peaks at approximately the same positions than for the radiative decay rate. The radiative yield $\eta$ in the absence of intrinsic losses for resonances corresponding to the first five peaks, starting from the longer wavelength, are $\sim 0.63,0.75,0.56,0.34$, and 0.24 .

We can relate the position of the peaks with the plasmon excitations of an infinite thin film of same thickness. Such films contain two plasmonic modes [11], named the short and long range plasmon according to their propagation length. They are also called antisymmetric and symmetric modes according to the symmetry of the magnetic field. The positions of the $\Gamma_{R}$ and $\Gamma_{\mathrm{NR}}$ maxima for the flat disk in Figs. 1(c) and 1(d), approximately agree with the expression $R=m \lambda_{\mathrm{sp}}^{\mathrm{sr}} / 2$, where $\lambda_{\mathrm{sp}}^{\mathrm{sr}}$ is the wavelength of the short range plasmon and $m$ is an integer that serves to classify the order of the resonance. Such simple correlation does not exist between the position of the peaks and the long range plasmon wavelength $\lambda_{\mathrm{sp}}^{\mathrm{lr}}$ for $m>1$, suggesting that only the short range surface plasmon is efficiently excited. Furthermore, looking at the near fields in the proximity of the disk at the five main $\Gamma_{R} / \Gamma_{0}$ maxima, the symmetry of the different field components (not shown) approximately corresponds to the symmetry of the short range plasmon. In summary, the disk appears to be a cavity into which the
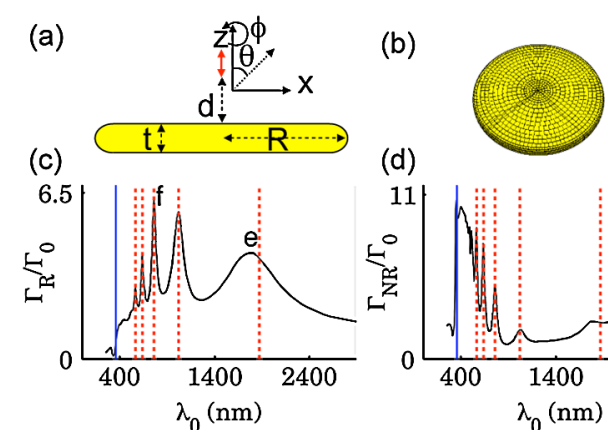

(e1)
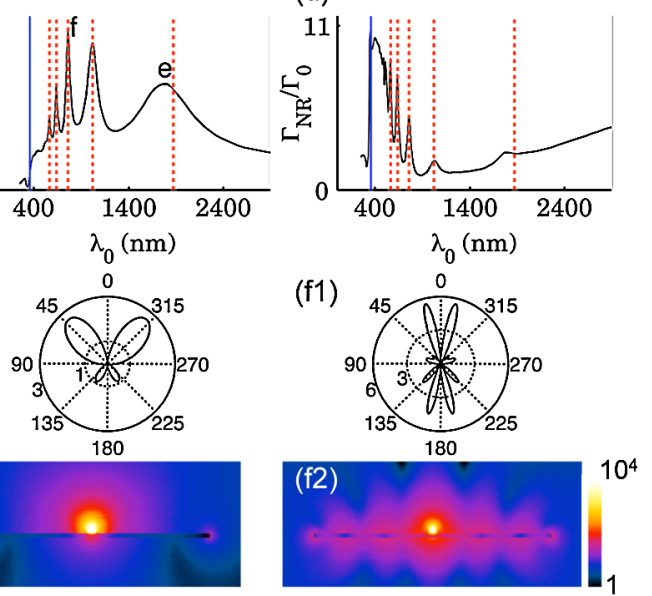

FIG. 1 (color online). (a) Schematical side view and coordinate axis for the flat disk, where the angles $\theta, \phi$ define the emission direction. The structure is rotationally symmetric with respect to the $z$ axis, as stressed by the three-dimensional image in (b). (c) Radiative $\Gamma_{R} / \Gamma_{0}$ and (d) nonradiative $\Gamma_{\mathrm{NR}} / \Gamma_{0}$ change on the emission rate for a disk with $R=600 \mathrm{~nm}$ radius, $t=$ $20 \mathrm{~nm}$ thickness and quantum emitter oriented vertically along the $z$ axis and situated over the center of the structure, at $d=$ $20 \mathrm{~nm}$ from the top surface. The dashed red lines correspond to the excitation frequency at which $R=m \lambda_{\mathrm{sp}}^{\mathrm{sr}} / 2 ; m$ takes the integer values 1 to 5 , with 1 corresponding to the longer wavelength. The solid blue vertical line at $\lambda_{0}=358.3 \mathrm{~nm}$ marks the frequency at which $\operatorname{Re}\left[\epsilon_{\mathrm{Ag}}\right]=-\epsilon_{g}$, the value of the resonance for a vertical quantum emitter in front of a silver halfspace. (e1),(f1) show the directivity $D(\theta)$ for same conditions at the maxima labeled $e\left(\lambda_{0}=1763 \mathrm{~nm}\right)$ and $f\left(\lambda_{0}=759.9 \mathrm{~nm}\right)$ in (c). (e2),(f2) show the amplitude of the electric field module in the $x z$ plane, in logarithmic scale and arbitrary units, in the proximity of the disks for the same wavelengths.

quantum emitter excites short range surface plasmons $[14,22]$, which undergo reflection at the edges and interfere constructively for adequate resonant conditions. These resonances enhance both $\Gamma_{R}$ and $\Gamma_{\mathrm{NR}}$.

To analyze in more detail the role of short range surface plasmons in the decay rate, we consider again the case of an infinite silver film of $20 \mathrm{~nm}$ thickness. $\Gamma_{T}$ is proportional to the LDOS $\rho_{z}^{e}$ component, which can be cast $[16,17,23]$ as an integral over contributions from plane waves of wave vector $\left(\mathbf{K}, k_{g}^{z}\right)$, with $\mathbf{K}$ perpendicular and $k_{\mathbf{g}}^{\mathbf{z}}$ parallel to the $z$ axis, $\mathbf{K}$ real, and $k_{g}^{2}=\left(k_{g}^{z}\right)^{2}+|\mathbf{K}|^{2}$. More exactly $\rho_{z}^{e}=$ $\rho_{z}^{e 0}\left[1+\int_{0}^{\infty} \xi_{z z}(K) d K\right]$, where $\rho_{z}^{e 0}$ is the value in the absence of the film, $K=|\mathbf{K}|, \quad \xi_{z z}(K)=3 /\left(2 k_{g}^{3}\right) \times$ $\operatorname{Im}\left[\mathbf{i} K^{3} r_{F}(K) e^{2 \mathbf{i} k_{g}^{z} d} / k_{g}^{z}\right], k_{g}=2 \pi \sqrt{\epsilon_{g}} / \lambda_{0}, \operatorname{Im}[]$ refers to the imaginary part and $r_{F}(K)$ is the Fresnel reflection factor of the film for a $p$-polarized plane wave described by $K$. We first show in Fig. 2(a) that the wavelength dependence for an infinite film roughly corresponds to the spectrum for the flat disks, apart from a modulation in the latter due to 
cavity resonances. The similarities justify the use of the thin film to study the coupling between the plasmons and the quantum emitter. By plotting $\xi_{z z}(K)$ in Fig. 2(b) for $\lambda_{0}=750 \mathrm{~nm}$ and the nonpropagative contributions $(K>$ $k_{g}$ ) associated with plasmons, we find two clear peaks corresponding to the two branches of the surface plasmon. The long range surface plasmon contribution yields a narrow peak in $K$ space close to $K=k_{g}$ whereas the short range surface plasmon yields a broader peak for $K \sim$ $1.28 \mathrm{k}_{\mathrm{g}}$. The absolute maximum corresponds to the long range plasmon, but the contribution to the integral $\int_{0}^{\infty} \xi_{z z}(K) d K$ from the broad peak associated with the short range plasmon predominates. This is the case for the five main $\Gamma_{R} / \Gamma_{0}$ maxima in Fig. 1(c). The key aspect is thus that to describe the excitation of the plasmonic modes, the broad $K$ spectrum of the dipolar, pointlike source has to be considered. A significant fraction of the source $K$ components excites the short range plasmon.

We consider next the directivity $D(\theta, \phi), D(\theta)$ in our rotationally symmetric case. Figures $1(\mathrm{e} 1)$ and 1(f1) show such results for two different orders $m$. The maxima next to the $z$ axis $(\theta=0,180)$ become narrower and closer to the axis for larger $m$, as the disk emitting area increases when measured in units of wavelength. The disk emission patterns can also present additional maxima for larger values of $|\theta|,|\pi-\theta|$, which diminishes the maximum directivity. The directivity $\sim 5.5$ in Fig. 1(f1) is comparable to values for Yagi-Uda optical nanoantennas [4,5].

The disks allow for a good understanding of the physics involved, but the directivity and enhancement of the decay rates $\Gamma_{T} / \Gamma_{0}$ shown so far are moderate. For comparison, we have obtained similar values for a $300 \mathrm{~nm}$ radius silver sphere in glass, with an emission predominantly directed in the backwards $90^{\circ}<\theta<270^{\circ}$ direction. For single photon sources, it is desirable to find structures that further increase the Purcell factor, while simultaneously achieving a radiative yield and directivity as large as possible. To this aim, we first draw inspiration from the lightning rod effect and the cascade enhancement [24]. We add at the center of
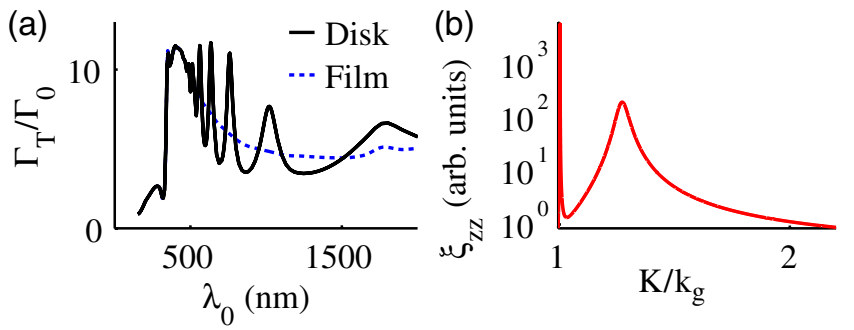

FIG. 2 (color online). (a) Purcell factor $\Gamma_{T} / \Gamma_{0}$ for the $R=$ $600 \mathrm{~nm}$ flat disk and for an infinite film of same thickness $t=$ $20 \mathrm{~nm}$ and distance $d=20 \mathrm{~nm}$ to the emitter. (b) $\xi_{z z}(K)$, function that describe the LDOS in $K$ space as $\rho_{z}^{e}=\rho_{z}^{e 0}[1+$ $\left.\int_{0}^{\infty} \xi_{z z}(K) d K\right]$, for a $20 \mathrm{~nm}$ thick infinite film, $\lambda_{0}=750 \mathrm{~nm}$ and $d=20 \mathrm{~nm}$. The narrow and broad peaks correspond to the long and short range plasmons, respectively. the disk a $20 \mathrm{~nm}$ high protuberance that ends in a semisphere of $10 \mathrm{~nm}$ radius, with the quantum emitter at $d=$ $10 \mathrm{~nm}$ directly over the upper point of the protuberance [see Fig. 3(a)]. The optical nanoantenna is otherwise identical to the previous disk. Values of the Purcell factor larger than 500 are possible [Fig. 3(a)]. The radiative rate $\Gamma_{R}$ also increases strongly, but the radiative yield $\eta$ at the $\Gamma_{R}$ maximum is only $\eta \sim 0.17$ [Fig. 3(b)]. Using $d=20 \mathrm{~nm}$ affects $\eta$ less than a $\sim 30 \%$ in the peak region $\sim 580-660 \mathrm{~nm}$ and at the same time reduces the maximum $\Gamma_{T}$ more than sixfold. Looking at the directivity in the presence of the protuberance [Fig. 3(c)], for $\lambda_{0}=$ $603.4 \mathrm{~nm}$ near the $\Gamma_{R}$ maximum, it again presents a narrow lobe at small values of $|\theta|,|180-\theta|$ and significant additional maxima for other angles, for a maximum value $\sim 4.2$. The directivity over all $\theta$ is very similar to the results (not shown) for the flat disk at the same wavelength $\lambda_{0}=$ $603.4 \mathrm{~nm}$ and distance $d=10 \mathrm{~nm}$.

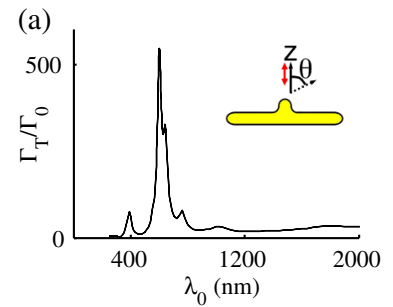

(b)

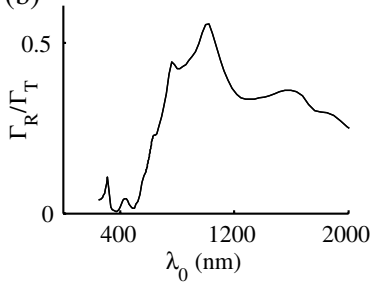

(c)

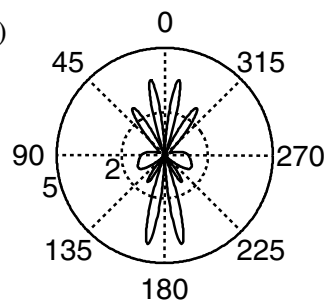

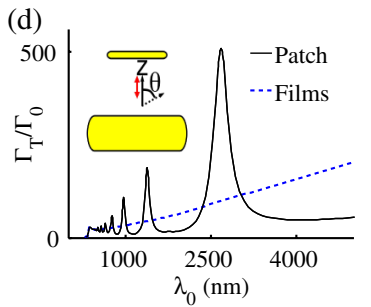

(e)

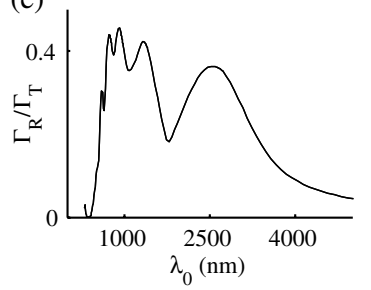

(f)

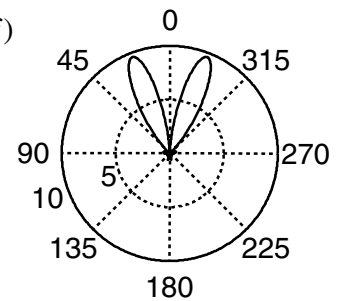

FIG. 3 (color online). (a),(d) Purcell factor $\Gamma_{T} / \Gamma_{0}$, (b), (e) radiative yield $\eta=\Gamma_{R} / \Gamma_{T}$ in the absence of intrinsic losses and (c),(f) directivity $D(\theta)$ for: (a)-(c) a $600 \mathrm{~nm}$ radius and $20 \mathrm{~nm}$ thick silver disk with a $20 \mathrm{~nm}$ high protuberance at the symmetry axis, with a vertically oriented quantum emitter at $10 \mathrm{~nm}$ distance over the top of the protuberance; (d)-(f) The solid black line corresponds to a vertically oriented quantum emitter at $20 \mathrm{~nm}$ of the surface of two rotationally symmetric silver disks, one of radius $R=600 \mathrm{~nm}$ and thickness $t=20 \mathrm{~nm}$ and the second of $R=1000 \mathrm{~nm}$ and $t=100 \mathrm{~nm}$. The center of the two disks and the dipole are aligned along $z$. The dashed blue line in (d) corresponds to a similar scenario, but with infinite films of the same thickness instead of the disks. (c) corresponds to the main peak in (a), $\lambda_{0}=603.4 \mathrm{~nm}$, and (f) to the second larger peak in (d), $\lambda_{0}=1381 \mathrm{~nm}$. All structures are included in glass $\epsilon_{g}=2.25$ and the disks have rounded edges. 
We consider next the possibility to increase the Purcell factor by introducing an extra confinement of the fields in the vertical direction. We illustrate in Fig. 3(d) how inserting the dipole between two infinite films can already result in significant Purcell factors, $>30$ for $\lambda_{0}>900 \mathrm{~nm}$. The results were obtained using a formalism similar to the one used for the single film in Fig. 2. The following step is to introduce cavity resonances by using finite disks instead of infinite films, in a structure similar to classical patch antennas [Figs. 3(d)-3(f)]. It consists of two silver disks inserted in glass, separated by a constant $40 \mathrm{~nm}$ distance and again rotationally symmetric with respect to the $z$ axis. The top disk has a radius $R=600 \mathrm{~nm}$ and thickness $t=20 \mathrm{~nm}$, while the bottom is significantly larger, with $R=1000 \mathrm{~nm}$ and $t=100 \mathrm{~nm}$. The vertically oriented quantum emitter occupies the center of the structure.

The decay rate continues to present clear resonance maxima, but the interaction between the disks [10] changes the characteristics of the plasmonic modes supported by the structure and the peaks positions are different from the single thin disk case. The clear maxima suggest an efficient reflection of the plasmons at the edges of the smaller disk. As a result of this strong reflection and the large field confinement, the Purcell factor at the strongest peak exceeds 500 with a radiative yield $\sim 0.36$. The emission pattern in Fig. 3(f) for the second largest peak $\lambda_{0}=$ $1381 \mathrm{~nm}$ demonstrates the possibility to obtain a maximum directivity close to 10 (9.6), benefiting from a considerable suppression of backwards emission. Looking into the collection efficiency $\beta$ for this peak, it is possible to capture $\sim 70 \%$ of the emitted photons using a spherical lens of numerical aperture $0.95\left(|\theta| \lesssim 40^{\circ}\right.$ in the glass) situated along the $z$ axis. As a reference, the value for a quantum emitter in homogeneous glass, without any optical antenna, is only $\sim 3.5 \%$ and $\sim 15 \%$ for the dipolar moment oriented parallel or perpendicular, respectively, to the $z$ axis. A resonant $300 \mathrm{~nm}$ radius silver sphere in similar conditions to the patch antenna results in $\beta \lessgtr 30 \%$. Together with the large directivity, the patch antenna peak at $\lambda_{0}=1381 \mathrm{~nm}$ is characterized by a full width at half maximum of about $90 \mathrm{~nm}$, a maximum Purcell factor $\Gamma_{T} / \Gamma_{0} \sim 190$, and a radiative yield $\eta \sim 0.42$.

In summary, the broad response in $K$ and frequency space makes plasmonic nanoantennas well suited for single photon emission. A very interesting feature of the plasmonic patch antenna is that it offers simultaneously a Purcell factor larger than 100 and a collection efficiency $\sim 70 \%$ for a lens of numerical aperture $<1$ over a broad spectral range. As the proposed structure simply consists of disks separated in the vertical $z$ dimension, emitting perpendicularly to the disk, it seems promising for experimental realization.

We acknowledge financial support from the ANR project PEEM-Plasmon, the project LETI-Plasmonics, the support of the RTRA Triangle de la Physique and the European project EQUIND.
*On leave from Institute of Radio Engineering and Electronics (Saratov Division), Russian Academy of Sciences, Zelyonaya 38, 410019 Saratov, Russia. jean-jacques.greffet@institutoptique.fr

[1] B. Lounis and M. Orrit, Rep. Prog. Phys. 68, 1129 (2005).

[2] I. Friedler, C. Sauvan, J. P. Hugonin, P. Lalanne, J. Claudon, and J. M. Gérard, Opt. Express 17, 2095 (2009).

[3] J.M. Gerard, B. Sermage, B. Gayral, B. Legrand, E. Costard, and V. Thierry-Mieg, Phys. Rev. Lett. 81, 1110 (1998).

[4] T. H. Taminiau, F. D. Stefani, and N. F. van Hulst, Opt. Express 16, 10858 (2008).

[5] H. F. Hofmann, T. Kosako, and Y. Kadoya, New J. Phys. 9, 217 (2007).

[6] T. H. Taminiau, F. D. Stefani, and N. F. van Hulst, New J. Phys. 10, 105005 (2008).

[7] O.L. Muskens, V. Giannini, J.A. Sánchez-Gil, and J. Gómez Rivas, Nano Lett. 7, 2871 (2007); L. Rogobete, F. Kaminski, M. Agio, and V. Sandoghdar, Opt. Lett. 32, 1623 (2007).

[8] C. A. Balanis, Antenna Theory. Analysis and Design (John Wiley \& Sons, Inc., Hoboken, 2005).

[9] C. Manolatou and F. Rana, IEEE J. Quantum Electron. 44, 435 (2008); A. Andronico, J. Claudon, J.-M. Gérard, V. Berger, and G. Leo, Opt. Lett. 33, 2416 (2008).

[10] G. Lévêque and O. J.F. Martin, Opt. Express 14, 9971 (2006).

[11] H. Raether, Surface Plasmons on Smooth and Rough Surfaces and on Gratings (Springer-Verlag, Berlin, Heidelberg, 1998).

[12] L. Novotny, Phys. Rev. Lett. 98, 266802 (2007); H. Ditlbacher, A. Hohenau, D. Wagner, U. Kreibig, M. Rogers, F. Hofer, F. R. Aussenegg, and J. R. Krenn, Phys. Rev. Lett. 95, 257403 (2005); J. Dorfmüller, R. Vogelgesang, R. T. Weitz, C. Rockstuhl, C. Etrich, T. Pertsch, F. Lederer, and K. Kern, Nano Lett. 9, 2372 (2009).

[13] Y. Kurokawa and H. T. Miyazaki, Phys. Rev. B 75, 035411 (2007).

[14] T. Søndergaard and S. I. Bozhevolnyi, Phys. Rev. B 75, 073402 (2007).

[15] J. Jung, T. Søndergaard, and S. I. Bozhevolnyi, Phys. Rev. B 79, 035401 (2009).

[16] L. Novotny and B. Hecht, Principles of Nano-Optics (Cambridge University Press, Cambridge, 2006).

[17] K. Joulain, R. Carminati, J.-P. Mulet, and J.-J. Greffet, Phys. Rev. B 68, 245405 (2003).

[18] S. Kühn, G. Mori, M. Agio, and V. Sandoghdar, Mol. Phys. 106, 893 (2008); H. Gersen, M. F. García-Parajó, L. Novotny, J. A. Veerman, L. Kuipers, and N. F. van Hulst, Phys. Rev. Lett. 85, 5312 (2000).

[19] E. Palik, Handbook of Optical Constants of Solids (Academic Press, San Diego, 1985).

[20] I. A. Larkin, M. I. Stockman, M. Achermann, and V.I. Klimov, Phys. Rev. B 69, 121403(R) (2004).

[21] T. Sannomiya, J. Vörös, and C. Hafner, J. Comput. Theor. Nanosci. 6, 749 (2009).

[22] E.S. Barnard, J.S. White, A. Chandran, and M.L. Brongersma, Opt. Express 16, 16529 (2008).

[23] G. W. Ford and W. H. Weber, Phys. Rep. 113, 195 (1984).

[24] J. Dai, F. Cajko, I. Tsukerman, and M. I. Stockman, Phys. Rev. B 77, 115419 (2008). 